Chronic Obstructive Pulmonary Diseases:

Journal of the COPD Foundation

COPD

FOUNDATION

\author{
Journal Club
}

\title{
Journal Club: Inhaled Corticosteroids- Refining Our Understanding of Their Role in Maintenance Treatment for COPD
}

\author{
Ron Balkissoon, MD, MSc, DIH, FRCPC ${ }^{1}$
}

\begin{abstract}
Abbreviations: Global initiative for chronic Obstructive Lung Disease, GOLD; forced expiratory volume in 1 second, FEV $\mathbf{1}$; inhaled corticosteroid, ICS; long-acting muscarinic antagonist, LAMA; long-acting beta2-agonists, LABA; chronic obstructive pulmonary disease, COPD

Citation: Balkissoon R. Journal club: Inhaled corticosteroids-refining our understanding of their role in maintenance treatment for COPD. Chronic Obstr Pulm Dis. 2017;4(2):162-167. doi: https://doi.org/10.15326/jcopdf.4.2.2017.0143
\end{abstract}

\section{Denver, Colorado}

Address correspondence to:

Ron Balkissoon, MD, MSc, DIH, FRCPC

balkissoonr@njhealth.org

\section{Keywords:}

chronic obstructive pulmonary disease; COPD; inhaled corticosteroids

\section{Introduction}

The most recent iteration of the Global initiative for chronic Obstructive Lung Disease (GOLD) Guidelines $(2017)^{1}$ has removed spirometry (forced expiratory volume in 1 second $\left[\mathrm{FEV}_{1}\right]$ from the algorithm for treatment recommendations and now determines treatment options based on the degree of symptoms and the frequency of exacerbations. In earlier iterations of the GOLD guidelines, inhaled corticosteroids (ICSs) were recommended for patients with either low lung function (FEV 1 less than 50\%) and/or for patients with 2 or more exacerbations or 1 hospitalization in the previous 12 months. The basis for using the cut-off of 2 or more exacerbations per year to define increased risk was partly predicated on the findings that the greatest benefit of ICSs was found for those that had 2 or more exacerbations per year. ${ }^{2}$ Subsequently, the long- acting muscarinic antagonist (LAMA) tiotropium was demonstrated to reduce exacerbations compared to long-acting beta2-agonists (LABAs). ${ }^{3-5}$ In the past few years there has been compelling evidence presented that ICSs can increase the risk of pneumonia in at least a subset of chronic obstructive pulmonary disease (COPD) patients. ${ }^{6-13}$ Further, there have been ongoing concerns about other deleterious effects of ICSs with respect to bone density, cataracts, oral thrush, and hoarseness such that the early enthusiasm for use of ICSs has become somewhat tempered. Indeed, the 2017 iteration of GOLD $^{1}$ has relegated ICS/LABA use to patients in Groups $C$ and D who do not show a reduction in exacerbations while on LAMAs or LABA/ LAMA.

The WISDOM trial ${ }^{14}$ had all patients $(n=2296)$ on ICS/LABA/LAMAs for 6 weeks and then withdrew ICS from 1 group and compared an ICS/LABA/LAMA combination versus a LABA/LAMA combination for 9 months. Patients entered in the trial only had to have had 1 exacerbation in the past 12 months. The WISDOM trial demonstrated no increased risk (non-inferiority) of exacerbations for 9 months following discontinuation of ICSs. These studies likely contributed to the latest GOLD recommendations for LABA/LAMA combination therapy being tried before ICS/LABA combinations for patients in Groups $C$ and $D$. The further rationale for use of LABA/LAMA over ICS/LABA is the reported increased risk of pneumonia related to ICS use for at least a sub-group of COPD patients.

The FLAME study ${ }^{15}$ ( $\mathrm{n}=3362$ ) compared once-daily LABA/LAMA indacaterol/glycopyrronium 110/50 $\mathrm{gg}$ with twice-daily LABA/ICS salmeterol/ fluticasone combination 50/500 $\mu \mathrm{g}$. The main result of the FLAME study was that indacaterol/glycopyrronium 110/50 $\mathrm{ug}$ was superior to the salmeterol/fluticasone combination $50 / 500 \mu \mathrm{g}$ in reducing the annual rate of moderate to severe exacerbations (0.98 versus 1.19; rate ratio, 0.83; 
95\% confidence interval [CI], 0.75 to $0.91 ; p<0.001$ ) and that there was a lower rate of pneumonia in the LABA/ LAMA group (3.2\%) versus the LABA/ICS group 4.8\% $(p=0.02)$. The results of these 2 studies undoubtedly informed the most recent recommendations found in GOLD 2017 related to LABA/LAMA combinations being the first choice compared to LABA/ICS combinations for patients in Groups $C$ and D. Both groups of investigators have performed post hoc analyses however, to examine whether there were any subgroups for whom LABA/ICS combinations might be selected before LABA/LAMA combinations. In this Journal Club we review the post hoc analyses of the FLAME study and the WISDOM trial that look at the role of peripheral blood eosinophil counts and frequency of exacerbations as factors that may identify a subgroup of patients that are more likely to benefit from ICS/LABA rather than LABA/LAMA combinations.

\section{Abstract 1 \\ Blood Eosinophil Count and Exacerbations in Severe Chronic Obstructive Pulmonary Disease After Withdrawal of Inhaled Corticosteroids: A Post-Hoc Analysis of the WISDOM Trial}

Watz H, Tetzlaff K, Wouters EF, et al. Lancet Respir

Med. 2016;4(5):390-398.

\section{BACKGROUND:}

Blood eosinophil counts might predict response to inhaled corticosteroids (ICS) in patients with chronic obstructive pulmonary disease (COPD) and a history of exacerbations. We used data from the WISDOM trial to assess whether patients with COPD with higher blood eosinophil counts would be more likely to have exacerbations if ICS treatment was withdrawn.

\section{METHODS:}

WISDOM was a 12-month, randomised, parallelgroup trial in which patients received $18 \mu \mathrm{g}$ tiotropium, 100 $\mathrm{gg}$ salmeterol, and $1000 \mu \mathrm{g}$ fluticasone propionate daily for 6 weeks and were then randomly assigned $(1: 1)$ electronically to receive either continued or reduced ICS over 12 weeks. We did a post-hoc analysis after complete ICS withdrawal (months 3-12) to compare rate of exacerbations and time to exacerbation outcomes on the basis of blood eosinophil subgroups of increasing cutoff levels. The WISDOM trial is registered at ClinicalTrials.gov, number NCT00975195.

\section{FINDINGS:}

In the 2296 patients receiving treatment after ICS withdrawal, moderate or severe exacerbation rate was higher in the ICS-withdrawal group versus the ICScontinuation group in patients with eosinophil counts (out of total white blood cell count) of $2 \%$ or greater (rate ratio 1.22 [95\% CI 1.02-1.48]), $4 \%$ or greater $(1.63[1 \cdot 19-2 \cdot 24])$, and $5 \%$ or greater $(1.82[1.20$ $2 \cdot 76])$. The increase in exacerbation rate became more pronounced as the eosinophil cutoff level rose, with significant treatment-by-subgroup interaction reached for $4 \%$ and $5 \%$ only. Similar results were seen with eosinophil cutoffs of 300 cells per $\mu \mathrm{L}$ and 400 cells per $\mu \mathrm{L}$, and mutually exclusive subgroups.

\section{INTERPRETATION:}

Blood eosinophil counts at screening were related to the exacerbation rate after complete ICS withdrawal in patients with severe to very severe COPD and a history of exacerbations. Our data suggest that counts of $4 \%$ or greater or 300 cells per $\mu \mathrm{L}$ or more might identify a deleterious effect of ICS withdrawal, an effect not seen in most patients with eosinophil counts below these thresholds.

\section{Comments}

The WISDOM trial enrolled individuals who had experienced 1 or more exacerbations in the previous 12 months and followed the groups for 9 months after steroids were withdrawn from 1 arm of the study. In review of the original paper and the post hoc studies, I could not find the total number of patients enrolled that had 2 or more exacerbations per year compared to 1 exacerbation. The fact that all participants were treated with fluticasone propionate $500 \mu \mathrm{g}$ bid for 6 weeks and then treated for another 3 months with either full or tapering doses provides a fairly substantial course of high dose ICSs for over 4 months following which they were followed for less than a year off of ICSs. Considering that the GOLD guidelines only suggest the use of inhaled steroids in patients that have had 2 or more exacerbations it would be very helpful to know the number of patients in this study that would have even normally been considered for treatment with 
ICSs. The WISDOM study also showed that there was a significant between group difference in the loss of lung function at week 52, (43 ml greater in the salmeterol/ tiotropium group $(p=0.001)$. The above post hoc analysis of the WISDOM trial data by Watz and colleagues demonstrated that for individuals with an eosinophil count greater than $4 \%$ or absolute count of 300 cells/ $\mu \mathrm{L}$ there was a significant reduction in exacerbations over the 9 months for those who remained on ICSs. The differences were even more pronounced when the cutoffs were $5 \%$ or 400 eosinophils/ $\mu \mathrm{L}$. This initial post hoc analysis did not analyze the frequency of exacerbations as a further defining factor with regard to increased risk of steroid withdrawal. The authors suggest that prospective studies are required to further delineate the utility of using peripheral blood eosinophils as a determinant for treatment with ICSs.

\section{Abstract 2 Blood Eosinophils and Response to Maintenance COPD Treatment: Data from the FLAME Trial}

Roche N, Chapman KR, Vogelmeier CF, et al. Am J Respir Crit Care Med. 2017; In press.

\section{RATIONALE:}

Post-hoc analyses suggest that blood eosinophils have potential as a predictive biomarker of inhaled corticosteroid efficacy in the management of chronic obstructive pulmonary disease.

\section{OBJECTIVES:}

We prospectively investigated the value of blood eosinophils as a predictor of responsiveness to an inhaled corticosteroid/long-acting $\beta 2$-agonist combination versus a long-acting $\beta 2$-agonist/ long-acting muscarinic antagonist combination for exacerbation prevention.

\section{METHODS:}

We conducted prespecified analyses of data from the FLAME study, which compared once-daily longacting $\beta 2$-agonist/long-acting muscarinic antagonist indacaterol/glycopyrronium $110 / 50 \mu \mathrm{g}$ with twicedaily long-acting $\beta 2$-agonist/inhaled corticosteroid salmeterol/fluticasone combination 50/500 $\mu$ in patients with $\geq 1$ exacerbation in the preceding year.
Subsequent post-hoc analyses were conducted to address further cut-offs and endpoints.

\section{MEASUREMENTS AND MAIN RESULTS:}

We compared treatment efficacy according to blood eosinophil percentage ( $<2 \%$ and $\geq 2 \%,<3 \%$ and $\geq 3 \%$, and $<5 \%$ and $\geq 5 \%$ ) and absolute blood eosinophil count ( $\leq 150$ cells $/ \mu l, 150$ to $<300$ cells $/ \mu l$, and $\geq 300$ cells $/ \mu l)$. Indacaterol/glycopyrronium was significantly superior to salmeterol/fluticasone for the prevention of exacerbations (all severities, or moderate or severe) in the $<2 \%, \geq 2 \%,<3 \%,<5 \%$ and $<150$ cells/ul subgroups, and at no cut-off was salmeterol/fluticasone superior to indacaterol/ glycopyrronium. Furthermore, the rate of moderate or severe exacerbations did not increase with increasing blood eosinophils. The incidence of pneumonia was higher in patients receiving salmeterol/fluticasone than indacaterol/glycopyrronium in both the $<2 \%$ and $\geq 2 \%$ subgroups.

\section{CONCLUSIONS:}

Our prospective analyses indicate that indacaterol/ glycopyrronium provides superior or similar benefits over salmeterol/fluticasone regardless of blood eosinophil levels in patients with COPD. Clinical trial registration available at www.clinicaltrials.gov, ID NCT01782326.

\section{Comments}

The FLAME trial also included COPD individuals who experienced 1 or more exacerbations in the previous 12 months. The authors reported that indacaterol/ glycopyronium significantly reduced exacerbations compared to fluticasone propionate/salmeterol 500/50 and demonstrated lower rates of pneumonia. It is worth noting that at study entry only about $20 \%$ or so of the patients in this trial had experienced 2 or more exacerbations in the previous 12 months and would have met the standard criteria for consideration of treatment with an ICS/LABA combination. Participants in this trial were not an ICS naïve population as substantial numbers, about $55 \%$, were on some form of ICS either as an ICS/LABA or ICS/LABA/LAMA combination prior to the beginning of the trial. There was only a 4-week washout period during which time patients were on tiotropium alone before randomization. The authors stratified by eosinophil level but they did not stratify 
by exacerbation frequency as Calverley and colleagues did in their post hoc analysis that is included in the same issue of the American Journal of Respiratory and Critical Care Medicine (See below). ${ }^{16}$ It is worth noting that both WISDOM and FLAME studies utilized the fluticasone/salmeterol dose of 500/50 rather than the $250 / 50$. Interestingly the $250 / 50$ dose is the only dose recommended in the United States for COPD patients as it appeared there was no compelling evidence that the 500/50 dose provided additional benefit in reducing exacerbations. There remain questions as to whether a lower dose or different formulations of ICSs may reduce the risk of pneumonia. ${ }^{10}$

\section{Letter to the Editor Eosinophilia, Frequent Exacerbations, and Steroid Response in Chronic Obstructive Pulmonary Disease}

Calverley PM, Tetzlaff K, Vogelmeier C, et al. Am J

Respir Crit Care Med. 2017; In press.

Letter to the Editor: (No abstract included)

\section{Comments}

While this is only a letter to the editor and there is no abstract, the relevance of the data presented in same March 2017 issue of the American Journal of Respiratory and Critical Care Medicine as the FLAME post hoc analysis is instructive. The WISDOM trial group further analyzed the data and showed that the findings were most pronounced in the groups who had 2 or more exacerbations per year and an eosinophil count of at least $300 / \mu \mathrm{L}$. This would certainly be consistent with the current recommendations that ICS/ LABA combinations are indicated for individuals with 2 or more exacerbations per year. The authors point out the limitations of a retrospective post-hoc analysis with relatively small sample sizes and the need to do further prospective trials to establish the validity of these cutoffs. With the limitations of their analysis being a post hoc analysis, the authors suggested that the combination of exacerbations of 2 or more per year and an elevated eosinophil count of 300 or more, identifies a subgroup that may potentially benefit from using an ICS/LABA combination compared to using a LABA/LAMA combination. For those with fewer exacerbations and lower eosinophil counts, a LABA/ LAMA combination may be an appropriate choice. The 2017 GOLD Guidelines do refer to the eosinophil counts as a factor to consider but suggests that it requires further study to decide how it may be useful in deciding between LAMA/LABA versus ICS/LABA combinations in COPD patients.

\section{Summary}

The pneumonia signal associated with ICS use has been convincingly demonstrated for a subset of patients that have COPD and frequent exacerbations. One should take this into consideration in determining whether ICS/LABA formulations are appropriate or relatively contraindicated. Nonetheless, the initial WISDOM trial and the FLAME trial results and subsequent assertions related to the relative benefits of LABA/ LAMA over ICS/LABA combinations in the COPD population need to be put in context with regard to the patient populations studied (many participants had less than 2 exacerbations), and the ICS used (fluticasone propionate $500 \mu \mathrm{g}$, the ICS formulation associated with the highest rate of pneumonia). To gain clarity for future guidelines, further studies are required to address the appropriate patient population where ICS/LABA use may be more appropriate and perhaps considered first line therapy over LABA/ LAMA combinations in patients who are being initially considered for maintenance therapy. Considering that there does not seem to be any significant benefit from the use of the 500/50 formulation of fluticasone/ salmeterol for reduction of exacerbations compared to the 250/50 formulation and the fact that most of the other ICS/LABA formulations are closer to the 250/50 fluticasone/salmeterol combination with regard to relative steroid dose, the trials should have an arm that uses the moderate dose ICS/LABA combinations. An instructive prospective trial would include patients with 2 or more exacerbations in the previous year and would randomize to ICS/LABA versus LABA/LAMA combinations and be stratified according to baseline blood eosinophil counts. The active treatment period should also be at least for 12 months. Patients with a history of recurrent pneumonia should be excluded. Given that these previous trials were performed with the high dose fluticasone ICS/LABA formulation it would perhaps be useful to compare the high dose 
ICS/LABA to moderate dose equivalent ICS/LABA formulations with regard to efficacy and safety in this high exacerbation/high eosinophil enriched cohort. We may find that, for at least some patients, the benefits may outweigh the risks. 


\section{References}

1. Vogelmeier CF, Criner GJ, Martinez FJ, et al. Global Strategy for the Diagnosis, Management, and Prevention of Chronic Obstructive Lung Disease 2017 report: GOLD Executive Summary. Arch Bronconeumol. 2017;53(3):128-149. doi: https://doi.org/10.1016/j.arbres.2017.02.001

2. Hurst JR, Vestbo J, Anzueto A, et al. Susceptibility to exacerbation in chronic obstructive pulmonary disease. $N$ Engl J Med. 2010; 363:1128-1138. doi: https://doi.org/10.1056/NEJMoa0909883

3. Vogelmeier C, Hederer B, Glaab T, et al. Tiotropium versus salmeterol for the prevention of exacerbations of COPD. N Engl J Med. 2011; 364:1093-1103.

doi: https://doi.org/10.1056/NEJMoa 1008378

4. Tashkin DP, Celli B, Senn S, et al. A 4-year trial of tiotropium in chronic obstructive pulmonary disease. N Engl J Med. 2008; 359:1543-1554.

doi: https://doi.org/10.1056/NEJMoa0805800

5. Wedzicha JA, Calverley PMA, Seemungal TA, Hagan G, Ansari Z, Stockley RA. The prevention of chronic obstructive pulmonary disease exacerbations by salmeterol/fluticasone propionate or tiotropium bromide. Am J Respir Crit Care Med. 2008;177(1):1926. doi: https://doi.org/10.1164/rccm.200707-973OC

6. Calverley PM, Stockley RA, Seemungal TAR, et al. Reported pneumonia in patients with COPD: Findings from the INSPIRE study. Chest. 2011;139(3):505-512.

doi: https://doi.org/10.1378/chest.09-2992

7. Crim C, Calverley PMA, Anderson JA, et al. Pneumonia risk in COPD patients receiving inhaled corticosteroids alone or in combination: TORCH study results. Eur Respir J. 2009; 34(3): 641-647. doi: https://doi.org/10.1183/09031936.00193908

8. Crim C, Dransfield MT, Bourbeau J, et al. Pneumonia risk with inhaled fluticasone furoate and vilanterol compared with vilanterol alone in patients with COPD. Ann Am Thorac Soc. 2015; 12(1):27-34.

doi: https://doi.org/10.1513/AnnalsATS.201409-413OC

9. Stanbrook MB. Review: In COPD, fluticasone or budesonide increases serious pneumonia but not mortality. Ann Intern Med. 2014;161(4): JC8.

doi: https://doi.org/10.7326/0003-4819-161-4-201408190-02008

10. Suissa S, Coulombe J, Ernst P. Discontinuation of inhaled corticosteroids in COPD and the risk reduction of pneumonia. Chest. 2015; 148(5):1177-1183.

doi: https://doi.org/10.1378/chest.15-0627

11. Suissa S, Patenaude V, Lapi F, Ernst P. Inhaled corticosteroids in COPD and the risk of serious pneumonia. Thorax. 2013; 68(11):1029-1036.

doi: https://doi.org/10.1136/thoraxjnl-2012-202872
12. White P. Inhaled fluticasone and budesonide increased the risk of serious pneumonia in COPD. Evid Based Med. 2014;19(3):116. doi: https://doi.org/10.1136/eb-2013-101695

13. Yawn BP, Li YF, Tian HJ, Zhang J, Arcona S, Kahler KH. Inhaled corticosteroid use in patients with chronic obstructive pulmonary disease and the risk of pneumonia: a retrospective claims data analysis. Int J Chron Obstruct Pulmon Dis. 2013; 8: 295-304. doi: https://doi.org/10.2147/COPD.S42366

14. Magnussen H, Disse B, Rodriguez-Roisin R, et al. Withdrawal of inhaled glucocorticoids and exacerbations of COPD. $N$ Engl J Med. 2014; 371(14): 1285-1294. doi: https://doi.org/10.1056/ NEJMoa1407154

15. Wedzicha JA, Banerji D, Chapman KR, et al. Indacaterolglycopyrronium versus salmeterol-fluticasone for COPD. N Engl J Med. 2016; 374:2222-2234.

doi: https://doi.org/10.1056/NEJMoa 1516385

16. Calverley PM, Tetzlaff K, Vogelmeier YF, et al. Eosinophilia, frequent exacerbations and steroid response in chronic obstructive pulmonary disease. Am J Respir Crit Care Med. 2017; In press. doi: https://doi.org/10.1164/rccm.201612-2525LE 